Conclusions Due to HIV + people enjoying a longer life expectancy, and an insufficient drop in incidence, HIV prevalence will rise as a result of ART. Modelling suggests that even small increases in risky sexual behaviour will lead to further substantial increases in HIV prevalence. Policy makers are urged to continue promoting sex education, and be prepared for a higher than previously suggested number of HIV+ people in need of treatment.

\section{P1-S6.52 IS PEP A MISUSED THERAPY? CROSS SECTIONAL PEP STUDY IN SEX WORKER OUTREACH PROGRAM CLINIC}

\section{doi:10.1136/sextrans-2011-050108.276}

\author{
1,2Jane Mungai, 1,2 Marion Kiguoya, ${ }^{1,2}$ Charles Wachihi, 1,2Joshua Kimani, \\ 1,2Festus Kiogora, ${ }^{1,2}$ James Mwanjewe, 1,2 Lawrence Gelmon. ' University of Nairobi; \\ ${ }^{2}$ University of Manitoba STI Collaboration
}

Background Post-exposure prophylaxis (PEP) is a short-term antiretroviral (ARV) treatment that reduces the likelihood of HIV infection after exposure to HIV-infected blood or sexual contact with an HIV-positive person. We are able to offer PEP mediations in our SWOP clinics to clients reporting high risk exposure. Normally ARVs for PEP are given within $72 \mathrm{~h}$ of exposure for a period of 4 weeks. Our objective was to assess whether the clients had already established STIs prior administration of PEP. The clients were aged between 19 and 49 years with each having an average of 9 men clients per day.

Methods A cross sectional study was conducted on 91 female sex workers who came to the clinic for PEP in the period September 2009 to July 2010. The female sex workers were first given counselling, completed a standard questionnaire before having PEP administered. The samples taken included blood for HIV Elisa, high vaginal swab for Gonorrhoea Culture on Thayer martin media and Gram stain smear for Bacteria vaginosis, presence of spermatozoa and white blood cells.

Results The clients were all HIV seronegative. $76 \%$ of the women had come for PEP for the first time, 21\% for the second time and 3\% for the third time. $80 \%$ of the patients reported condom burst, $8.6 \%$ were as a result of rape or coerced sex, while $3.2 \%$ reported client refused condom use. However $73 \%$ smears of the women did not have spermatozoa. Overall $9 \%$ of the patients were GC positive but $3.2 \%$ had GC and spermatozoa while $6.6 \%$ had GC without spermatozoa. Trichomonas prevalence was $4.3 \%$ but all these patients did not have spermatozoa. $38 \%$ had a WBC count of over $6-30$ field on gram stain Conclusion: The presence of high white cell count at the time of seeking PEP may indicate a pre-existing infection, hence presence of underlying high risk behaviour. Moreover presence of GC and TV without spermatozoa may also indicate exposure longer reported. There is need to educate sex workers on proper use of PEP and to maintain low risk behaviour. We also need to understand the decision making process of sex workers in choosing post-exposure prophylaxis and any barriers that may contribute to delays in seeking PEP.

\section{P1-S6.53 ANTIRETROVIRAL THERAPY REDUCES HIV TRANSMISSION IN DISCORDANT COUPLES IN NORTHERN MALAWI}

\section{doi:10.1136/sextrans-2011-050108.277}

${ }^{1} \mathrm{~J}$ Glynn, ${ }^{1} \mathrm{~A}$ Price, ${ }^{1} \mathrm{~S}$ Floyd, ${ }^{1} \mathrm{~A}$ Molesworth, ${ }^{2} \mathrm{~N}$ Kayuni, ${ }^{2} \mathrm{M}$ Chihana, ${ }^{2} \mathrm{~V}$ Nyirenda, ${ }^{1} \mathrm{~A}$ Crampin, ${ }^{1} \mathrm{~N}$ French. ${ }^{1}$ London School of Hygiene \& Tropical Medicine, Chilumba, Malawi; ${ }^{2}$ Karonga Prevention Study, Malawi

Background Antiretroviral therapy (ART) reduces viral load and has been suggested as a prevention measure to reduce transmission. However there are concerns that this may be counterbalanced to some extent by increased sexual activity associated with improved health and decreased condom use. The impact of ART on rates of HIV-transmission was investigated within HIV discordant couples in rural, Northern Malawi.

Methods The study was conducted as part of the Karonga Prevention Study. A demographic surveillance system was started in 2001 and annual home-based HIV sero-surveys added from 2007, with individual counselling and consent. From the first round of the serosurvey, 201 HIV discordant couples (147 male positive, female negative; 54 male negative, female positive) were identified for whom follow-up HIV data were available in the negative partner. Free ART is available at local health facilities and ART use was determined by linkage of data to a clinic based cohort and selfreport. Patients attend ART clinics with a guardian who may be the spouse, and are counselled about the need for safer sex. For this analysis participant exposure to ART was defined as having started ART prior to study entry, and follow-up was censored at time of last observation, HIV seroconversion, last negative HIV test, death, departure or dissolution of marriage. Follow-up with re-testing is ongoing.

Results So far there are 249 and 99 person-years of follow-up in HIV negative women and men, respectively. 55 of the 201 positive partners had commenced ART at entry and within these couples no HIV transmission occurred over a 3-year follow-up period. Among 146 discordant couples without ART the rate of transmission to females was 5.2 / 100 person years (95\% CI 2.7 to 10.5) and to males $1.3 / 100$ person years ( $95 \%$ CI 0.18 to 9.4 ).

Conclusions In HIV discordant couples ART is associated with reduced rates of HIV transmission within partnerships.

\section{Epidemiology poster session 6: Preventive intervention: Circumcision}

\section{P1-S6.54 MEDICAL MALE CIRCUMCISION MAY BE PROTECTIVE OF UROGENITAL MYCOPLASMA GENITALIUM INFECTION: RESULTS FROM A RANDOMISED TRIAL IN KISUMU, KENYA}

doi:10.1136/sextrans-2011-050108.278

${ }^{1} \mathrm{~S}$ Mehta, ${ }^{2} \mathrm{C}$ Gaydos, ${ }^{3} \mathrm{I}$ Maclean, ${ }^{4} \mathrm{E}$ Odoyo-June, ${ }^{3} \mathrm{~S}$ Moses, ${ }^{4} \mathrm{R}$ Murugu, ${ }^{4} \mathrm{~L}$ Agunda, ${ }^{4} \mathrm{E}$ Nyagaya, ${ }^{2} \mathrm{~N}$ Quinn, ${ }^{1} \mathrm{R}$ Bailey. ${ }^{1}$ University of Illinois, Chicago, Chicago, USA; ${ }^{2}$ The Johns Hopkins University School of Medicine, USA; ${ }^{3}$ University of Manitoba, Winnipeg, Canada; ${ }^{4}$ UNIM Project, Kenya

Background We determined the prevalence of Mycoplasma genitalium (MG) and whether this was associated with circumcision status, among men enrolled in the randomised trial of medical male circumcision (MMC) to prevent HIV acquisition in Kisumu, Kenya.

Methods MG was detected in first void urine (FVU) by APTIMA transcription mediated amplification (TMA) assay. FVU and urethral swabs were assessed for Neissseria gonorrhoeae (NG) and Chlamydia trachomatis(CT) by PCR, and for Trichomonas vaginalis (TV) by culture. HSV-2 detection was by IgG ELISA. Personal interview assessed socio-demographic and behavioural risks. All men underwent standardised medical history and physical examination.

Results July through September 2010, 52 (9.9\%; 95\% CI: 7.3 to $12.4 \%)$ MG infections were detected among 526 men. The prevalence of NG (1.4\%) and TV (2.7\%) did not differ by MG status. CT prevalence was $5.8 \%$ among MG-infected men, and $0.8 \%$ among $\mathrm{MG}$-uninfected men $(\mathrm{p}=0.02)$. CT infection at enrolment was more prevalent among MG infected men $(27 \%$ vs $9 \%$ uninfected, $\mathrm{p}=0.005)$; NG and TV at enrolment were not associated with MG. Current urethral discharge symptoms and exam findings did not vary by $M G$ status ( $1.9 \%$ positive vs $1.5 \%$ negative, $p=0.80)$, but 